Proc. of The Fifth Intl. Conf. On Advances in Applied Science and Environmental Engineering - ASEE 2016

Copyright (C) Institute of Research Engineers and Doctors, USA .All rights reserved.

ISBN: 978-1-63248-086-6 doi: 10.15224/ 978-1-63248-086-6-05

\title{
Generalized Fock space for the Airy operator
}

Akram Nemri, Fethi Soltani and Abo-el-nour Abd-alla

\begin{abstract}
In this work, we introduce the Fock space $F_{v}(C)$ associated to the Airy operator, and we establish Heisenberg-type uncertainty principle for this space. Next, we give an application of the theory of extremal function and reproducing kernel of Hilbert space, to establish the extremal function associated to a bounded linear operator $T: F_{v}(C) \rightarrow H$, where $H$ be a Hilbert space. Finally, we come up with some results regarding the extremal functions, when $T$ is the difference operator and the Dunkl-difference operator, respectively.
\end{abstract}

Keywords-component, Airy-type Fock space, Tikhonov regularization, Heisenberg-type uncertainty principle.

\section{I- Introduction}

The study of several generalizations of the classical Fock spaces has a long and rich history in many different settings (see for instance [11], [12]). In this work, we will try to generalize Airy-type Fock space, to establish Heisenberg-type uncertainty principle for this space; and to give an application of the theory of reproducing kernels to the Tikhonov regularization on this generalized Fock space.

The generalized Airy operator (or hyper-Bessel operator [14, 15]) is third singular differential operator given by

$$
L_{v}:=\frac{d^{3}}{d x^{3}}+\frac{3 v}{x} \frac{d^{2}}{d x^{2}}-\frac{3 v}{x^{2}} \frac{d}{d x},
$$

where $v$ is a nonnegative real number. When $v=0$, this operator becomes the third derivative operator for which some analysis were studied by Widder [18] and for some special value of $v$ the operator $L_{v}$ appeared as a radial part of the generalized Airy equation of a nonlinear diffusion type partial differential equation in $R^{n}$. Recently, in a nice and long paper, Cholewinski and Reneke [2] studied and extended, for the operator $L_{v}$, the well known theory related to some singular differential operator of second order for which the literature is extensive.

Akram Nemri

Department of Mathematics, Faculty of Science, Jazan University, Jazan, Saudi Arabia

Fethi Soltani

Department of Mathematics, Faculty of Science, Jazan University Jazan, Saudi Arabia

Abo-el-nour Abd-alla

Department of Mathematics, Faculty of Science, Jazan University Jazan, Saudi Arabia
Next, Fitouhi et al. [7, 8] established a harmonic analysis related to this operator for examples the eigenfunctions, the generalized translation, the Fourier-Airy transform, the heat equation, the heat polynomials, the transmutation operators, ... During the last years, the Airy operator have gained considerable interest in various field of mathematics [3-6], [1315] and in certain parts of quantum mechanics [1]. The results of this work will be useful when discussing the Fock space associated to this operator.

This space is the background of some applications in this contribution. Especially,

- we study the Airy operator and its adjoint operator on the Airy-type Fock space.

- We establish Heisenberg-type uncertainty principle for the Airy-type Fock space.

- We give an application of the theory of extremal function and reproducing kernel of Hilbert space, to establish the extremal function associated to a bounded linear operator $T: F_{v}(C) \rightarrow H$, where $H$ be a Hilbert space.

- We come up with some results regarding the extremal functions associated to the following operators:

- The difference operator $T f(z):=\frac{1}{z^{3}}(f(z)-f(0))$.

- And the Dunkl-difference operator $T f(z):=\frac{1}{2 z^{3}}(f(z)-f(-z))$

The contents of the paper are as follows. In Section 2, we introduce the Airy-type Fock space $F_{v}(C)$. In Section 3, we establish Heisenberg-type uncertainty principle for this space. In Section 4, we give an application of the theory of reproducing kernels to the Tikhonov regularization problem for bounded linear operator $T: F_{v}(C) \rightarrow H$, where $H$ be a Hilbert space. Next, we come up with some results regarding the Tikhonov regularization problem for the difference operator and the Dunkl-difference operator, respectively.

\section{II- Airy-type Fock space}

Let $z \in C$ and $\omega_{k}=e^{2 i \pi \frac{k-1}{3}}, k=1,2,3$. A function $u(z)$ is called 3-even if $u\left(\omega_{k} z\right)=u(z)$.

For $\lambda \in C$, the initial problem $L_{v} u(z)=\lambda^{3} u(z)$, with $u(0)=1$ and $u^{(k)}(0)=0, k=1,2$ admits a unique analytic solution on $C$, which will be denoted by $G_{v}(\lambda z)$ and expanded in power series as

$G_{v}(\lambda z)=\sum_{n=0}^{\infty} \frac{(\lambda z)^{3 n}}{\alpha_{3 n}(v)}$, where 
$\alpha_{3 n}(v)=3^{3 n} n ! \frac{\Gamma\left(n+\frac{1}{3}\right) \Gamma\left(n+v+\frac{2}{3}\right)}{\Gamma\left(\frac{1}{3}\right) \Gamma\left(v+\frac{2}{3}\right)}$

The function $G_{v}(\lambda z)$ is 3-even and defined as the hypergeometric function (see [2]),

$G_{v}(\lambda z)={ }_{0} F_{2}\left[\frac{1}{3}, v+\frac{2}{3} \mid\left(\frac{\lambda z}{3}\right)^{3}\right]$.

In particular $\left|G_{v}(\lambda z)\right| \leq e^{|\lambda \|| z \mid}$ and

$G_{0}(\lambda z)=\cos _{3}(-\lambda z)=\sum_{n=0}^{\infty} \frac{(\lambda z)^{3 n}}{(3 n) !}$.

We define the Airy-type Fock space $F_{v}(C)$ as the prehilbertian space of 3-even entire functions $f(z)=\sum_{n=0}^{\infty} a_{n} z^{3 n}$ on $\mathrm{C}$ such that

$\|f\|_{F_{V}(C)}^{2}=\sum_{n=0}^{\infty}\left|a_{n}\right|^{2} \alpha_{3 n}(v) \prec \infty$.

Let $f$ and $g$ be in $F_{v}(C)$ such that $f(z)=\sum_{n=0}^{\infty} a_{n} z^{3 n}$ and $g(z)=\sum_{n=0}^{\infty} b_{n} z^{3 n}$, the inner product in $F_{v}(C)$ is given by $\langle f, g\rangle_{F_{v}(C)}:=\sum_{n=0}^{\infty} a_{n} \overline{b_{n}} \alpha_{3 n}(v)$.

The space $F_{v}(C)$ satisfies the following properties.

(i) The function $K_{v}$ given by $K_{v}(w, z)=G_{v}(\bar{w} z)$ is a reproducing kernel for the space $F_{v}(C)$.

(ii) If $f \in F_{v}(C)$, then $|f(z)| \leq e^{\frac{|z|^{2}}{2}}\|f\|_{F_{v}(C)}, z \in C$.

(iii) The space $F_{v}(C)$ equipped with the inner product $\langle.,\rangle_{F_{v}(C)}$ is a Hilbert space; and the set $\left\{\frac{z^{3 n}}{\sqrt{\alpha_{3 n}(v)}}\right\}_{n \in N}$ forms an Hilbert's basis for the space $F_{v}(C)$.

\section{III- Heisenberg-type uncertainty principle}

We consider the following lemmas.

Lemma 3.1. Let $L_{v}: F_{v}(C) \rightarrow F_{v}(C)$, then its adjoint operator $L_{v}^{*}$ is given by $L_{v}^{*} f(z)=z^{3} f(z)$.
Lemma 3.2. $\left[L_{v}, L_{v}^{*}\right]:=L_{v} L_{v}^{*}-L_{v}^{*} L_{v}=(6+9 v) \mathrm{I}+\mathrm{B}_{v}$, where

$\mathrm{B}_{v} f(z)=9 z^{2} \frac{d^{2}}{d z^{2}} f(z)+18(1+v) z \frac{d}{d z} f(z)$.

Lemma 3.3. Let $F_{v}(C)$. Then

$$
\left\|L_{v}^{*} f\right\|_{F_{v}(C)}^{2}=\left\|L_{v} f\right\|_{F_{v}(C)}^{2}+(6+9 v)\|f\|_{F_{v}(C)}^{2}+\left\langle f, \mathrm{~B}_{v} f\right\rangle_{F_{v}(C)}
$$

By applying Lemma 3.1, Lemma 3.2, Lemma 3.3 and the following theorem.

Theorem 3.4. (See $[9,10]$ ). Let $A$ and $B$ be self adjoint operators on a Hilbert space $H$. Then we have

$$
\|(A-a) f\|_{H}\|(B-b) f\|_{H} \geq \frac{1}{2}\left|\langle[A, B] f, f\rangle_{H}\right|,
$$

for all $f \in \operatorname{Dom}(A B) \cap \operatorname{Dom}(B A)$, and all $a, b \in R$.

We obtain the following Heisenberg-type uncertainty principle for the Airy-type Fock space $F_{v}(C)$.

Theorem 3.5. Let $f \in F_{v}(C)$. For all $a, b \in R$, we have $\left\|\left(L_{v}+z^{3}-a\right) f\right\|_{F_{v}(C)}\left\|\left(L_{v}-z^{3}+i b\right) f\right\|_{F_{v}(C)} \geq \mathrm{A}_{v} f$,

where

$$
\mathrm{A}_{v} f=\left|(6+9 v)\|f\|_{F_{v}(C)}^{2}+\left\langle\mathrm{B}_{v} f, f\right\rangle_{F_{v}(C)}\right| .
$$

This uncertainty principle can be written as the following form.

Theorem 3.6. Let $f \in F_{v}(C)$. Then

$$
\Delta_{v}^{+}(f) \Delta_{v}^{-}(f) \geq\|f\|_{F_{v}(C)}^{2}\left(\mathrm{~A}_{v} f\right)^{2}
$$

where

$$
\Delta_{v}^{ \pm}(f)=\|f\|_{F_{v}(C)}^{2}\left\|\left(L_{v} \pm z^{3}\right) f\right\|_{F_{v}(C)}^{2}-\left|\left\langle\left(L_{v} \pm z^{3}\right) f, f\right\rangle_{F_{v}(C)}\right|^{2} .
$$

\section{IV- Extremal functions on $F_{v}(C)$}

Let $\lambda \succ 0$ and let $T: F_{v}(C) \rightarrow H$ be a bounded linear operator from $F_{v}(C)$ into a Hilbert space $H$. We denote by $\langle f, g\rangle_{L, \lambda}$ the inner product defined on the space $F_{v}(C)$ by

$$
\langle f, g\rangle_{L, \lambda}:=\lambda\langle f, g\rangle_{F_{v}(C)}+\langle T f, T g\rangle_{H} \text {. }
$$

By using the theory reproducing kernels of Hilbert space and building on the ideas of Saitoh [16,17] we establish the extremal function associated to the operator $T$ on the Airy-type Fock space $F_{v}\left(C^{d}\right)$. 
Theorem 4.1. Let $\lambda \succ 0$. The Fock space $\left(F_{v}(C),\langle.,\rangle_{T, \lambda}\right)$ possesses a reproducing kernel $K_{T, \lambda}(w, z) ; w, z \in C$, which satisfying the equation

$\left(\lambda I+T^{*} T\right) K_{T, \lambda}(w,)=.K_{v}(w,$.$) . Moreover the kernel$ $K_{T, \lambda}(w, z)$ satisfies the following properties.

(i) $\left\|K_{T, \lambda}(w, .)\right\|_{F_{v}(C)} \leq \frac{e^{\frac{|w|^{2}}{2}}}{\lambda}$,

(ii) $\left\|T K_{T, \lambda}(w, .)\right\|_{H} \leq \frac{e^{\frac{|w|^{2}}{2}}}{\sqrt{\lambda}}$,

(iii) $\left\|T^{*} T K_{T, \lambda}(w, .)\right\|_{F_{v}(C)} \leq e^{\frac{|w|^{2}}{2}}$.

From this theorem we obtain the main result of this section .

Theorem 4.2. For any $h \in H$ and for any $\lambda \succ 0$, there exists a unique function $f_{\lambda, h}^{*}$, where the infimum

$\inf _{f \in F_{v}(C)}\left(\lambda\|f\|_{F_{v}(C)}^{2}+\|h-T f\|_{H}^{2}\right)$ is attained.

Moreover, the extremal function $f_{\lambda, h}^{*}$ is given by

$f_{\lambda, h}^{*}(w)=\left\langle h, L K_{T, \lambda}(w, .)\right\rangle_{H}$ and satisfies the inequality

$\left|f_{\lambda, h}^{*}(w)\right| \leq\|h\|_{H} \frac{e^{\frac{|w|^{2}}{2}}}{\sqrt{\lambda}}$.

Application 4.3. Let $H$ be the prehilbertian space of 3even entire functions $f(z)=\sum_{n=0}^{\infty} a_{n} z^{3 n}$ on $\mathrm{C}$ such that

$\|f\|_{H}^{2}=\sum_{n=0}^{\infty}\left|a_{n}\right|^{2} \alpha_{3 n+3}(v) \prec \infty$. The space $H$ is a Hilbert space with Hilbert's basis $\left\{\frac{z^{3 n}}{\sqrt{\alpha_{3 n+3}(v)}}\right\}_{n \in N}$ and reproducing kernel $S_{v}(w, z)=\sum_{n=0}^{\infty} \frac{(\bar{w} z)^{3 n}}{\alpha_{3 n+3}(v)}$.

(i) Let $T$ be the difference operator defined on $F_{v}(C)$ by $T f(z):=\frac{1}{z^{3}}(f(z)-f(0))$.

The operator $T$ maps continuously from $F_{v}(C)$ into $H$, and we have

$K_{T, \lambda}(w, z)=\frac{1}{\lambda}+\frac{1}{\lambda+1}\left(G_{v}(\bar{w} z)-1\right)$,
$T K_{T, \lambda}(w,).(z)=\frac{1}{\lambda+1} \frac{G_{v}(\bar{w} z)-1}{z^{3}}$,

and $f_{\lambda, h}^{*}(w)=\frac{1}{\lambda+1} w^{3} h(w)$.

(ii) Let $T$ be the Dunkl-difference operator defined on

$$
F_{v}(C) \text { by } T f(z):=\frac{1}{2 z^{3}}(f(z)-f(-z)) \text {. }
$$

The operator $T$ maps continuously from $F_{v}(C)$ into $H$, and we have

$$
\begin{aligned}
& K_{T, \lambda}(w, z)=\frac{1}{\lambda} \sum_{n=0}^{\infty} \frac{(\bar{w} z)^{6 n}}{\alpha_{6 n}(v)}+\frac{1}{\lambda+1} \sum_{n=0}^{\infty} \frac{(\bar{w} z)^{6 n+3}}{\alpha_{6 n+3}(v)}, \\
& T K_{T, \lambda}(w, .)(z)=\frac{1}{\lambda+1} \sum_{n=0}^{\infty} \frac{(\bar{w})^{6 n+3} z^{6 n}}{\alpha_{6 n+3}(v)}, \\
& \text { and } f_{\lambda, h}^{*}(w)=\frac{1}{2(\lambda+1)} w^{3}(h(w)+h(-w)) .
\end{aligned}
$$

\section{Acknowledgment}

This work was supported by the Deanship of the Scientific Research of Jazan University, Saudi Arabia (Project $\mathrm{N}^{\circ}$ 3261-6-36).

\section{References}

[1] M.S. Ben Hammouda and A. Nemri, Polynomial expansions for solutions of Higher-order Bessel heat equation in quantum calculus, Fract. Cal. Appl. Anal. 10(1) (2007) 3958.

[2] F.M. Cholewinski and J.A. Reneke, The generalized Airy diffusion equation, Electron. J. Differential Equations 87 (2003) $1-64$.

[3] I. Dimovski, Operational calculus for a class of differential operators, C.R. Acad. Bulg. Sci. 19 (1966) 1111-1114.

[4] I. Dimovski, Foundations of operational calculi for the Bessel-type differential operators, Serdica. Bulg. Math. Publ-s 1 (1975) 51-63.

[5] I. Dimovski and V. Kiryakova, On an integral transformation, due to N. Obrechkoff, Lecture Notes in Math. 798 (1980) 141-147.

[6] I. Dimovski, V. Kiryakova, The Obrechkoff, integral transform: properties and relation to a generalized fractional calculus. Num. Funct. Anal. Optim. 21(1-2) (2000) 121-144.

[7] A. Fitouhi, N.H. Mahmoud and S.A. Ould Ahmed Mahmoud, Polynomial expansions for solutions of higher-order Bessel heat equations, J. Math. Anal. Appl. 206 (1997) 155167.

[8] A. Fitouhi, M.S. Ben Hammouda and W. Binous, On a third singular differential operator and transmutation, Far East J. Math. Sci. 21(3) (2006) 303-329. 
Proc. of The Fifth Intl. Conf. On Advances in Applied Science and Environmental Engineering - ASEE 2016

Copyright (C) Institute of Research Engineers and Doctors, USA .All rights reserved.

ISBN: 978-1-63248-086-6 doi: 10.15224/ 978-1-63248-086-6-05

[9] G. Folland, Harmonic analysis on phase space, Annals of

Mathematics Studies 122, Princeton University Press,

Princeton, New Jersey, 1989.

[10] K. Gröchenig, Foundations of time-frequency analysis, Birkhauser, Boston, 2001.

[11] K. Gröchening and J. Toft, The range of localization operators and lifting theorems for modulation and BargmannFock spaces, Trans. Amer. Math. Soc. 365(8) (2013) 44754496.

[12] J. Hong and O. Yacobi, Polynomial representations and categorifications of Fock space, Algebr. Represent. Theory 16(5) (2013) 1273-1311.

[13] V. Kiryakova, Generalized fractional calculus and Applications. Longman, Harlow; John Wiley, N. York (1994).

[14] V. Kiryakova, Obrechkoff integral transform and hyperBessel operators via G-function and fractional calculus approach. Global J. Pure Appl. Math. 1(3) (2005) 321-341.

[15] M.I. Klyuchantsev, Singular differential operators with $r$ 1 parameters and Bessel functions of vector index, Seberian Math. J. 24 (1983) 353-366.

[16] S. Saitoh, Hilbert spaces induced by Hilbert space valued functions, Proc. Amer. Math. Soc. 89 (1983) 74-78.

[17] S. Saitoh, Best approximation, Tikhonov regularization and reproducing kernels, Kodai Math. J. 28 (2005) 359-367.

[18] D.V. Widder, The Airy transform, Amer. Math. Monthly 86 (1979) 271-277. 